\title{
Un análisis crítico del concepto de resiliencia en psicología
}

\author{
Julio Alfonso Piña López \\ Programa de Salud Institucional. Universidad de Sonora (México).
}

\begin{abstract}
Resumen: Se analiza el concepto de resiliencia en psicología, poniendo especial énfasis en autores iberoamericanos que lo utilizan en sus investigaciones. Se demuestra cómo, una vez que se parte de una confusión lógica, de ésta se desprende una conceptual, de la que a su vez se desprende una metodológica, para concluir con una de interpretación. Una cadena de confusiones que al final del camino nos permiten justificar por qué el de resiliencia es un concepto que no es ni útil ni pertinente para la psicología.

Palabras Clave: Resiliencia, Psicología, Confusión Lógica, Confusión Conceptual.

Title: A critical analysis of the concept of resilience in psychology.

Abstract: The concept of resilience in the psychology is analyzed, with special emphasis in Ibero-American authors who used it in their research. I argue how, once the authors' part of a logical confusion, this one become in a conceptual confusion, which in turn follows a methodological, concluding with one of interpretation confusions. A successive chain of confusions which allows justifying why resilience is a concept that is neither helpful nor relevant to psychology.

Key Words: Resilience, Psychology, Logical Confusion, Conceptual Confusion.
\end{abstract}

La teoría científica de lo psicológico, como lenguaje técnico que describe las formas de las regularidades fenoménicas, desempeña el papel definitorio de la disciplina [...] la teoría se justifica en el mundo ordinario de lo psicológico, se compara con otras opciones epistémicos y lógicas, permite distinguir los conceptos psicológicos de los conceptos prestados por otras disciplinas, delimita las formas de colaboración con otras disciplinas, señala como se aplica el conocimiento psicológico a través de las interdisciplinas y desmitifica la patologización de la vida psicológica restaurando su calidad interpersonal y, por consiguiente, de capacidad social y compromiso moral correspondiente (Ribes, 2010; p. 63).

\section{Introducción}

La materia prima de la psicología, asegura Ribes (2010), está constituida por términos del lenguaje ordinario que tienen que ver con el decir, el sentir y el hacer de las personas comportándose en lo individual; destacan, entre otros, atender, percibir, hablar, pensar, razonar y recordar, así como fenómenos que incluyen a las emociones, el desarrollo, la personalidad, las competencias y los motivos (Ribes, 2004, 2010). Son términos que formarán parte de un tipo particular de teoría, sí, y sólo sí, antes se cumple con un paso intermedio que va del lenguaje de la historia natural de los fenómenos (como objetos concretos) tal y como se nos presentan en la vida cotidiana, al lenguaje teórico (como objetos conceptuales) de la disciplina (Ribes, 2011).

Puesto que los términos que provienen del lenguaje ordinario cobran sentido como prácticas sociales e interpersonales que tratan con la fenomenología "cruda" de lo psicológico, de acuerdo con el autor su función es una: la de la comunicación entre personas, identificando cómo la conducta de una persona afecta a la de otra mediante el lenguaje (Figura 1). Por su parte, en el lenguaje técnico cumplen con una función distinta: la de describir y designar. Esto es así en

* Dirección para correspondencia [Correspondence address]:

Guillermo Prieto No, 18. Colonia Constitución. Hermosillo, Sonora, C.P. 83150 (México). E-mail: pina.lopez.julio@gmail.com razón de que en el lenguaje ordinario los términos tienen tantos usos y significados como sean los contextos y circunstancias en que se les emplea, mientras que en el lenguaje técnico son unívocos y tienen un único significado cuando se les emplea como conceptos en una teoría (Ribes, 1990).

Dicho lo anterior, un psicólogo no puede tomar prestados los términos del lenguaje ordinario y hacer uso de los mismos como si fuesen términos técnicos. Al hacerlo está violentando un principio elemental, frecuentemente pasado por alto por el colectivo de psicólogos. Sobre el respecto, Ribes (1990) señala lo siguiente; citaremos por su importancia en extenso:

En conclusión, el análisis conceptual del lenguaje ordinario no constituye en sí una teoría psicológica, pero es indispensable para su formulación. Su utilidad radica en que, en primer lugar, permite identificar los errores y confusiones categoriales provenientes de la transmutación del lenguaje ordinario en lenguaje técnico, y a que, en segundo lugar, contribuye a deslindar el terreno de los eventos psicológicos cotidianos en sus diversos niveles de significado funcional, condición sine qua non para formular una taxonomía y lenguaje técnico específicos y adecuados a un análisis científico del comportamiento (Ribes, 1990; p. 20).

Es a partir de estas breves consideraciones en torno a las diferencias entre los lenguajes ordinario y técnico que se propone analizar un concepto que desde los años setenta del pasado siglo ha pasado a ocupar un lugar casi privilegiado en la psicología; nos referimos al de resiliencia. No se trata de un concepto cualquiera, por cuanto, y en principio, del mismo no podría siquiera decirse que tiene que ver con la fenomenología de lo psicológico, mucho menos que se distinga por su precisión y claridad al momento de definirlo.

De hecho, el lector podrá constatar que el de resiliencia ha adquirido un estatuto especial como una especie de concepto comodín y multiusos, al que inclusive se le confunde con otros conceptos o expresiones, como los de empatía, sentido del humor, afrontamiento, autoeficacia, perseverancia, competencia, religiosidad, optimismo, tenacidad, control personal, personalidad "resistente", etcétera. Incluimos aquí a autores que han justificado sus trabajos en un análisis con- 
ceptual del término (i.e., Earvolino-Ramirez, 2007; Fernández-Lansac y Crespo, 2011; Garcia-Dia, DiNapoli, GarciaOna, Jakoboswki, \& O’Flagerty, 2013; Windle, 2011), así como también a quienes lo usan rutinariamente en sus investigaciones.

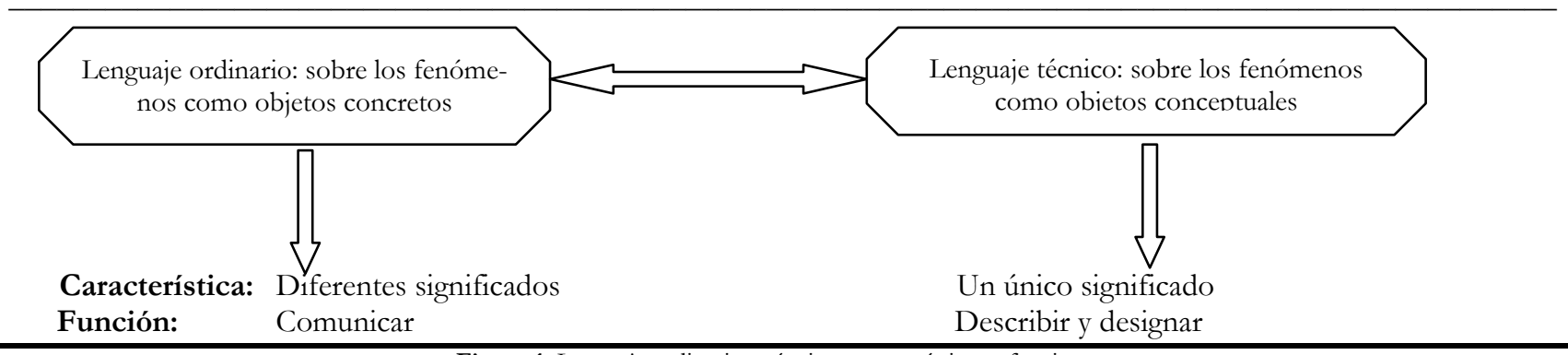

Figura 1. Lenguaje ordinario y técnico: características y funciones.

\section{El concepto de resiliencia en el lenguaje ordinario}

Resiliencia, en el lenguaje ordinario, es un verbo que proviene del latín resilio, resilire, que significa saltar hacia atrás, rebotar (Real Academia Española, 2011). Como se observa en la Tabla 1, este término se ha utilizado en diferentes disciplinas, incluidas la medicina (Cornejo, 2010) y la psicología. En esta última, y amparados en toda clase de justificaciones, lo mismo se asegura que como concepto es un término sugerente e interesante que precisa de una mayor clarificación
(Becoña, 2006); que si bien no es un concepto homogéneo, sirve para explicar algo (Vinaccia, Quiceno y Moreno-San Pedro, 2007); que uno de los problemas fundamentales en la investigación es su definición, no obstante existe consenso general que implica adaptación efectiva (Gaxiola, 2013); finalmente, que ante la diversidad de definiciones, es difícil pensar en una concepción única y hay que proponer una definición alternativa (González-Arratia, Valdez, Oudhof y González, 2012).

Tabla 1. El concepto de resiliencia en diferentes disciplinas e ingenierías.

\begin{tabular}{|c|c|}
\hline Disciplinas e ingenierías & Definiciones $^{a}$ \\
\hline Física & Capacidad de un material de recobrar su forma original luego de someterse a altas presiones. \\
\hline Ingeniería: Civil & $\begin{array}{l}\text { Magnitud que cuantifica la cantidad de energía por unidad de volumen que almacena un material al defor- } \\
\text { marse elásticamente debido a una tensión aplicada. }\end{array}$ \\
\hline Ingeniería: Metalurgia & Característica de los materiales de contraerse, dilatarse y recuperar su estructura interna. \\
\hline Ecología & Capacidad de las comunidades de soportar perturbaciones. \\
\hline Sociología & Capacidad que tienen los grupos para sobreponerse a los resultados adversos. \\
\hline Derecho & $\begin{array}{l}\text { Capacidad de las personas, dentro del marco general de los derechos humanos, de recuperar su estado origi- } \\
\text { nal de libertad, igualdad, inocencia, etcétera. }\end{array}$ \\
\hline Psicología & Capacidad humana de asumir con flexibilidad situaciones límite y sobreponerse a ellas. \\
\hline
\end{tabular}

Conocidas estas variadas justificaciones, los psicólogos, en particular en los países iberoamericanos:

1. Diseñan ex profeso o adaptan instrumentos de medida elaborados en otros países (Gaxiola, Frías, Hurtado, Salcido y Figueroa, 2011; Rodríguez, Pereyra, Gil, Jofré, de Bortoli y Labiano, 2009; Serrano-Parra, Garrido-Abejar, Notario-Pacheco, Bartolomé-Gutiérrez, Solera-Martínez y Martínez-Vizcaíno, 2012).

2. Con esos instrumentos realizan investigaciones sobre los problemas del desarrollo, la convivencia social, la educación o la salud (Gaxiola, González, Contreras y Gaxiola, 2012; Gaxiola, Pérez y González, 2013; GonzálezArratia, Reyes, Valdez y González, 2011; Quiceno, Vinaccia, Barrera, Latorre, Molina y Zubieta, 2013), y

3. Hay incluso quienes concluyen que existe una teoría científica en ciernes sobre la resiliencia (Becoña, 2006).

En suma, que se utilice el concepto, que se diseñen y/o adapten instrumentos, que se realice investigación y que se interpreten los datos para justificar su pertinencia y poder explicativo/predictivo, es un asunto que finalmente dependerá de si se cumplen o no con varios requisitos, uno de los cuales, por una petición elemental de principio, tiene que ver con su definición. Lo que pretendemos demostrar es que un término ajeno a la psicología, que posteriormente es mal definido como concepto - consecuencia de una confusión lógica primaria en torno a su origen-, a su vez acarrea una serie de confusiones metodológicas y de interpretación de los hallazgos que se obtienen al conducir una investigación.

\section{El concepto de resiliencia y las confusiones en psi- cología}

Una confusión lógica tiene lugar cuando se da por cierta la existencia de un fenómeno, como es el caso de la resiliencia, de igual manera que se habla de atender, percibir, pensar, razonar, recordar, etcétera; adicionalmente, cuando se asegu- 
ra que se trata de un fenómeno psicológico. Aun cuando el término haya aparecido primero en la literatura que se inscribe en el campo de la salud mental (Saavedra y Villalta, 2008) y que al día de hoy se haya expandido a campos como los del desarrollo, la personalidad, la educación y la salud, principalmente (Becoña, 2006; Fernández-Lansac y Crespo, 2011; Gaxiola et al., 2012; Windle, 2011), entre los diferentes autores hay preguntas que no han sido respondidas satisfactoriamente; mención especial merecen:

1. ¿Es la resiliencia un proceso o un resultado?

2. ¿Qué se quiere decir con resiliencia, o sea, es un atributo de la persona?

3. ¿Tiene unas bases biológicas plenamente identificadas?

4. ¿El ser o hacer resiliente se construye a lo largo del proceso de desarrollo?

5. ¿O bien, radica en el sistema de relaciones, esto es, como interacción de la persona con objetos, eventos u otras personas en los ambientes físico-químico, ecológico y/o social?

Para el común denominador de los psicólogos, la resiliencia se concibe como una respuesta adaptativa cuando una persona enfrenta condiciones adversas o de riesgo (Tabla 2), aun cuando no se ha precisado si finalmente se trata de: a) un proceso o un resultado; b) un atributo de la persona, en el sentido de que, inherente y consustancial a su existencia como ser biológico y social, hay alguna disposición hacia la resiliencia (Gaxiola et al., 2012), que está ahí, latente, a la espera de ser activada por alguna fuerza interna $y / 0$ externa; c) si es un concepto relativo a la reactividad, es decir, a la manera en que se responde a esas fuerzas externas que se conciben como adversas y potencialmente dañinas, perturbadoras, generadoras de estrés..., o d) si es consecuencia de la interacción de la persona con el ambiente en sus distintas modalidades.

Tabla 2. Definiciones sobre resiliencia en la psicología, por orden alfabético del(os) autor(es).

\begin{tabular}{|c|c|}
\hline Autor(es) & Definiciones \\
\hline Castillo y Haz (2003; p. 106). & $\begin{array}{l}{[\ldots] \text { se usará el término de resiliencia para describir los } \text { mecanismos }^{\text {a }} \text { psicológi- } \text {... }} \\
\text { cos e interrelacionales de las personas que han sido capaces de enfrentar esta ad- } \\
\text { versidad o evento traumático durante su infancia }[\ldots] \text {. }\end{array}$ \\
\hline Cornejo (2010; p. 5). & $\begin{array}{l}\text { La resiliencia en tanto cualidad de la personalidad, es la capacidad de los indi- } \\
\text { viduos, grupos o colectivos para afrontar diferentes tipos de adversidades y ha- } \\
\text { ciendo uso de recursos personales y sociales, asumir acciones resueltas para lograr } \\
\text { éxitos, auto o socio-realización y transformar dicha realidad. }\end{array}$ \\
\hline Gaxiola y Frías (2007; p. 222). & $\begin{array}{l}\text { La resiliencia se define como la capacidad para presentar respuestas adaptati- } \\
\text { vas ante condiciones adversas. }\end{array}$ \\
\hline Gaxiola, Frías, Hurtado, Salcido y Figueroa (2011; p. 74). & $\begin{array}{l}\text { La resiliencia es una inferencia basada en las diferencias individuales respecto a la } \\
\text { respuesta al estrés o adversidad }[\ldots] \text {; por tal motivo, constituye una variable del } \\
\text { orden latente }[\ldots] \text { que puede ser inferida a partir de indicadores observados rela- } \\
\text { tivos a los modos disposicionales }[\ldots] \text {, esto es, la forma como reacciona la per- } \\
\text { sona ante las adversidades que atraviesa. }\end{array}$ \\
\hline González-Arratia, Valdez y González (2011; p. 160). & $\begin{array}{l}\text { La comprensión conceptual }[. . .] \text { y empírica de la resiliencia nos ha llevado a con- } \\
\text { siderar que existen dos componentes generales: afectivo y cognitivo. Afectivo } \\
\text { en el sentido de los sentimientos y emociones que despierta una situación ad- } \\
\text { versa y el cognitivo consta de las percepciones del individuo sobre la situación } \\
\text { en concreto. }\end{array}$ \\
\hline $\begin{array}{l}\text { González-Arratia, Valdez, Oudhof y González (2012; p. } \\
\text { 50). }\end{array}$ & $\begin{array}{l}{[\ldots] \text { en esta investigación se entiende que es el resultado de la combinación o in- }} \\
\text { teracción entre los atributos del individuo (internos) y los propios de su am- } \\
\text { biente familiar, social y cultural (externos) que lo posibilitan para superar el ries- } \\
\text { go y la adversidad de forma constructiva. }\end{array}$ \\
\hline Ruiz y López (2012; pp. 2-3). & $\begin{array}{l}\text { En línea con esto, se acepta que la resiliencia no es un proceso extraordinario y se } \\
\text { asume que surge en el día a día, a partir de los recursos humanos de carácter } \\
\text { normativo, afectando a los individuos, a sus familias y a sus comunidades. Esta } \\
\text { visión de la resiliencia como proceso que surge de lo ordinario, de lo cotidiano } \\
\text { (frente a la noción de que se trata de una cualidad extraordinaria), implica una } \\
\text { perspectiva cultural puesto que, si se entiende como proceso que surge de lo } \\
\text { cotidiano, es indesligable de la influencia de los elementos socioculturales y } \\
\text { contextuales entre los que se desenvuelve la persona [...]. }\end{array}$ \\
\hline
\end{tabular}

${ }^{a}$ Resaltado en negrita por el autor del presente trabajo.

Cuando Gaxiola et al. (2011) señalan que el de resiliencia es un constructo inferido, ${ }^{1}$ sería equivalente a decir que es

${ }^{1}$ Los autores se refieren a que al concepto le subyace una serie de atributos individuales que han de ser descubiertos a través de la especificación puntual de diversas conductas, lo que le confiere el carácter de variable latente, tal y un constructo hipotético que propicia que se incurra en una causa falsa o, como elegantemente describe García-Cadena (2009), en una falacia post hoc ergo Procter hoc, que conduce a

como se le concibe en el contexto del modelamiento con ecuaciones estructurales. 
un error metodológico en su medición. Éste tiene que ver con la supuesta existencia de un factor especial subyacente, la capacidad de resiliencia, que en apariencia se distribuye normalmente en la población, de la misma manera que ocurre con la inteligencia (Ibáñez, 1992) y la personalidad (Ribes, 1990), por mencionar dos ejemplos conspicuos.

Ahora bien, aceptando sin conceder que ese factor subyacente existiera y que se concluyera que el de resiliencia es un concepto relativo a la capacidad de las personas para presentar respuestas adaptativas ante la adversidad (Gaxiola, 2013; Gaxiola y Frías, 2007; Noriega, Angulo y Hernández, 2011), el concepto así definido formaría parte de las palabras o expresiones que se agrupan o clasifican como capacidades, esto es, que existe la certeza de que algo es o será el caso (Ryle, 1967): su relación transitaría del 0 (algo no es o no será el caso) al 1 (algo es o será el caso). Un ejemplo sobre el respecto se tiene cuando se le pregunta a una persona: ¿eres capaz de resolver el problema de cuánto es $2+2$ ? En este caso ser capaz implica que la persona estaría en condiciones de resolverlo diciendo que $2+2$ es igual a 4 . En consecuencia, ser capaz describe la conducta en potencia (en tanto posibilidad), no en acto (Ribes, 2006). ${ }^{2}$

En tal virtud, no es un concepto del que se pueda decir que existe determinada probabilidad de que algo será o que se practicarán determinadas conductas, porque las palabras o expresiones de capacidades implican posibilidades. Así, ser resiliente en tanto se es capaz, no significaría otra cosa que estar en condiciones de hacer algo siempre y cuando se dé la oportunidad o que se configure una determinada circunstancia. Por consiguiente, si el concepto de resiliencia se aplica a capacidades, no se le puede igualar o hacer depender de conceptos tales como competencias, habilidades, autoeficacia, aprendizaje, afrontamiento y personalidad, según sugieren varios autores (véanse Becoña, 2006; Cornejo, 2010; Gaxiola et al., 2011; Gaxiola et al., 2012; González-Arratia et al., 2011; Saavedra y Villalta, 2008; Vinaccia et al., 2007).

Retomando a Ribes (2011), es necesario aclarar que, a propósito de los términos de capacidades, competencias y habilidades, aquél nos hace saber que ser capaz, ser competente y ser hábil es referirse a tres cosas distintas; se citará por su importancia de nuevo en extenso:

Sin embargo, ser capaz en este sentido no implica ser competente necesariamente, pues ser competente es siempre ser competente en algo o para algo [...] implica capacidad específica ya mostrada en acto en el pasado. Ser capaz no describe algo que ha hecho previamente o se hace, sino que significa estar en condiciones de hacer algo si de da la oportunidad o la circunstancia. Ser competente se refiere a que se puede hacer algo porque se ha hecho o porque se tiene conocimiento de lo que se tiene que hacer [...] Ser hábil es hacer algo que se ajusta a algo y, por consiguiente, implica una acción y un objeto sobre el que se ac-

\footnotetext{
2 Ser capaz difiere radicalmente de ser competente. Ser competente implica según Ribes (2006) tener la atribución para tratar de hacer algo porque ya se ha hecho antes en otra circunstancia; luego entonces, se ha hecho algo porque se conoce sobre algo y porque se dispone de los recursos conductuales para hacerlo en la forma de dominio de la técnica. Por tanto, si ser capaz implica posibilidad, ser competente implica probabilidad.
}

túa. No hay habilidades sin objetos de la acción, y sin ajuste de la acción a las características del objeto. Toda habilidad presupone siempre una técnica y su uso apropiado (Ribes, 2011; p. 37).

De esta cita textual se desprende que, contrario a lo que se plantea en la literatura referida en la Tabla 2, el de resiliencia como capacidad, aun cuando está "emparentado" con los conceptos de competencias y habilidades, no es equivalente a ninguno y mucho menos ambos se pueden reducir a aquél: en la medida en que pertenecen a categorías conceptuales distintas, su función lógica es también distinta. Es decir, cada uno sirve para predecir determinadas acciones (como conjuntos o colecciones de ocurrencias), que son de distintos tipos.

Si son capacidades en tanto que respuestas adaptativas - que es en donde se observa un mayor acuerdo entre los autores-, el de resiliencia no puede ser definido como proceso, contrario a lo expuesto por diversos autores (Becoña, 2006; Fernández-Lansac y Crespo, 2011; Ruiz y López, 2012), puesto que aquellas respuestas sirven para un fin particular, que es el de adaptación a las circunstancias sociales en las que se prescribe o demanda qué hacer.

Todavía más, si se aceptara el supuesto de la resiliencia como proceso, entonces la pregunta sería, ¿cómo se podrían diferenciar a los procesos psicológicos resilientes que supuestamente subyacen a la resiliencia como capacidad adaptativa en tanto resultado? Si según Roca (2001), hablar de procesos psicológicos es hacer referencia a fenómenos tales como el aprendizaje, el desarrollo y el pensamiento, entonces los procesos resilientes no podrían ser tomados como equivalentes de los actos o conductas que se consideran resilientes, o sea, las respuestas instrumentales con fines de adaptación, en tanto evidencia de una capacidad de resiliencia.

Pero, todavía más, si se aceptan los procesos psicológicos sugeridos por Roca, luego entonces, ¿a qué procesos psicológicos se apela para asegurar que la resiliencia es un proceso? En todo caso, ¿cómo se infieren esos procesos psicológicos? Para dar respuesta a ambas preguntas es necesario adentrarse en la revisión de algunos instrumentos de medida, cuyos autores en ocasiones de manera tácita, en otras explícitamente, defienden la existencia de un supuesto factor o dimensión subyacente al que denominan resiliencia.

\section{El concepto de resiliencia y su medición: ¿hay un factor o dimensión psicológica subyacente?}

Tal y como se resume en la Tabla 3, a juicio de algunos autores existen diversos factores o dimensiones que subyacen a la resiliencia, contenidos en diversos instrumentos de medida, tanto originales como adaptados de una lengua a otra. Lo que interesa de manera especial es hacer notar que, si presumiblemente existe la resiliencia, una pregunta elemental que habría que hacer sería la siguiente: ¿cuántos factores o dimensiones son necesarios para asegurar su existen- 
cia? Asimismo, ¿son todos factores o dimensiones pertene- cientes a la fenomenología de lo psicológico?

Tabla 3. Factores/dimensiones resilientes subyacentes y otros instrumentos de medida.

\begin{tabular}{|c|c|c|}
\hline Autor(es) & Instrumento(s) & Factores/dimensiones \\
\hline Gaxiola et al. (2011). & $\begin{array}{l}\text { Inventario de resiliencia (elabora- } \\
\text { ción propia). }\end{array}$ & $\begin{array}{l}24 \text { preguntas y } 10 \text { dimensiones originales, de las cuales quedaron siete: } \\
\text { 1. Actitud positiva. } \\
\text { 2. Sentido del humor. } \\
\text { 3. Perseverancia. } \\
\text { 4. Religiosidad. } \\
\text { 5. Autoeficacia. } \\
\text { 6. Optimismo. } \\
\text { 7. Orientación hacia la meta. }\end{array}$ \\
\hline $\begin{array}{l}\text { González-Arratia et al. } \\
\text { (2012). }\end{array}$ & $\begin{array}{l}\text { Cuestionario de resiliencia (elabora- } \\
\text { ción propia). }\end{array}$ & $\begin{array}{l}\text { 32 preguntas agrupadas en } 3 \text { dimensiones: } \\
\text { 1. Factores protectores internos: habilidades para la solución de problemas. } \\
\text { 2. Factores protectores externos: apoyo de la familia y otras personas signi- } \\
\text { ficativas. } \\
\text { 3. Empatía: altruismo y conducta prosocial. }\end{array}$ \\
\hline $\begin{array}{l}\text { Palomar, Matus y Victo } \\
\text { (2012). }\end{array}$ & $\begin{array}{l}\text { Escala de resiliencia de Connor \& } \\
\text { Davidson (2003). }\end{array}$ & $\begin{array}{l}\text { 31 preguntas y cuatro factores: } \\
\text { 1. Confianza en sí mismo. } \\
\text { 2. Competencia social. } \\
\text { 3. Fortaleza individual. } \\
\text { 4. Estructura personal. }\end{array}$ \\
\hline $\begin{array}{l}\text { Restrepo, Vinaccia y Qu } \\
\text { ceno (2011). }\end{array}$ & $\begin{array}{l}\text { Escala de resiliencia para adolescen- } \\
\text { tes (elaboración de Restrepo, 2009). }\end{array}$ & $\begin{array}{l}\text { 21 preguntas agrupadas en tres dimensiones: } \\
\text { 1. Búsqueda de novedad. } \\
\text { 2. Regulación emocional. } \\
\text { 3. Orientación positiva hacia el futuro. }\end{array}$ \\
\hline Rodríguez et al. (2009). & $\begin{array}{l}\text { Adaptación de la escala de resilien- } \\
\text { cia de Wagnild, \& Young (1993). }\end{array}$ & $\begin{array}{l}25 \text { preguntas originales agrupadas en dos factores: competencias personales } \\
\text { y aceptación de sí mismo y de la vida. Un análisis factorial exploratorio arro- } \\
\text { jó una estructura de tres factores: } \\
\text { 1. Capacidad de autoeficacia. } \\
\text { 2. Capacidad de propósito y sentido de vida. } \\
\text { 3. Evitación cognoscitiva. }\end{array}$ \\
\hline
\end{tabular}

Si se toma como ejemplo el instrumento diseñado por Gaxiola et al. (2011), el primero en la Tabla 3, desde la justificación del diseño y de la validación los autores incurren en graves errores lógicos, conceptuales, metodológicos y de interpretación. Así, señalan que:

La medición de la resiliencia requiere de tres elementos principales: la identificación de los riesgos ${ }^{3}$ a los que se encuentren sometidas las personas, la medición de sus características disposicionales individuales, y la evaluación de las competencias, entendidas como el alcance de logros o metas en tareas del desarrollo adecuadas a la edad, el sexo y la cultura de las personas [...] El conjunto de atributos personales cuya combinación permite superar los efectos nocivos de las situaciones de riesgo considera un conjunto de cualidades que pueden ser enmarcadas en diez dimensiones de carácter disposicional, a saber: afrontamiento, actitud positiva, sentido del humor, empatía, flexibilidad, perseverancia, religiosidad, autoeficacia, optimismo y orientación a la meta (Gaxiola et al., 2011; p. $75)$.

Más adelante, los autores al definir cada una de las dimensiones, señalan que:

\footnotetext{
${ }^{3}$ Las palabras en negrita son del autor del presente trabajo.
}

1. El afrontamiento ${ }^{4}$ comprende la tendencia a enfrentar los problemas y la búsqueda de apoyos necesarios en caso de requerirse (p. 75).

2. La actitud positiva abarca el enfoque positivo de la vida, la búsqueda de personas positivas, la disposición para el aprendizaje ante los problemas y el tratar de ser feliz a pesar de las contrariedades que se enfrentan cotidianamente (p. 75).

3. El sentido del humor es la disposición a sonreír, incluso ante los problemas o situaciones difíciles (p. 75).

4. La empatía se define como el entender y compartir los estados emocionales o los contextos de las demás personas [...] implica la tendencia del individuo (p. 76).

5. La flexibilidad incluye la aceptación de los problemas como parte normal de la vida y la posibilidad de adaptarse a las pérdidas mediante un enfoque positivo hacia las cosas... (p. 76).

6. La perseverancia se entiende como la persistencia del esfuerzo para lograr metas a pesar de las dificultades y la habilidad para reajustarlas... (p. 76).

7. La religiosidad es la tendencia a conceptuar los problemas que se enfrentan de acuerdo a las creencias religiosas que se tengan (p. 76).

\footnotetext{
${ }^{4}$ Las palabras subrayadas y en negrita son del autor del presente trabajo.
} 
8. La autoeficacia consiste en las creencias en las propias capacidades para organizar y ejecutar los cursos de acción requeridos que producirán determinados logros o resultados (pp. 76-77).

9. El optimismo es la tendencia de las personas a esperar resultados positivos y favorables en su vida (p. 77).

10. La orientación hacia la meta consiste en tener metas y aspiraciones de la vida y hacer lo necesario para lograrlas (p. 77).

Si se presume que existe un constructo llamado resiliencia que, según estos autores tiene que ver con competencias conductuales, entonces cuando se revisan y analizan estas 10 dimensiones, resulta que ninguna de ellas tiene absolutamente nada que ver con el concepto de competencias. Incluso más preocupante es que estos autores hayan justificado la pertinencia del concepto de resiliencia basándose en un concepto como el de competencias incorrectamente definido, con el que dieron vida a una diversidad de fenómenos a los que sin más se les atribuye el carácter de psicológicos: tratar de ser feliz, sentido del humor, empatía, religiosidad y optimismo, por mencionar a los más conspicuos.

Es decir, resulta que ahora esas 10 dimensiones recién mencionadas forman parte de la fenomenología de lo psicológico. Es importante que los autores del citado instrumento (Gaxiola et al., 2011) tengan en cuenta que tratar de ser feliz, sentido del humor y optimismo, por ejemplo, podrían eventualmente formar parte de lo que Ryle (1967) dio en llamar la emotividad, que incluye a las motivaciones, los estados de ánimo, las conmociones y los sentimientos. Los tres primeros se conciben formando parte de las categorías conceptuales relativas a propensiones o inclinaciones, en tanto que el último forma parte de la de acontecimientos. ${ }^{5}$

No son, por tanto, disposiciones competenciales subyacentes, las que darían cuenta de la resiliencia, puesto que "tratar de ser feliz", "reír" y "ser optimista" son cosas distintas; de acuerdo con Ryle (1967), tratar de ser feliz implica una inclinación o propensión a, mientras que reír y ser optimista implican acontecimientos, esto es, sentimientos como ocurrencias o conductas particulares. De ahí que, en el sentido estricto de la palabra, reír y ser optimista no son dispo-

\footnotetext{
${ }^{5}$ En el marco de la propuesta de Ryle, el autor hace una distinción entre eventos psicológicos que pueden ser definidos y agrupados en distintas categorías conceptuales (como eventos disposicionales, en tanto conjuntos o colecciones de ocurrencias) y aquellos que pueden ser definidos como respuestas o conductas instrumentales. Pensar, por ejemplo, es un concepto disposicional que refiere una relación, pues cuando se piensa no se habla de una respuesta o conducta particular, sino de diversas respuestas o conductas implicadas en una circunstancia y respecto de eventos especiales. Así, cuando se dice, Juan está pensando, se dice que está pensando con relación a algo o alguien en una circunstancia particular (un examen). Por otro lado, los acontecimientos, se refieren y aplican a casos, ocurrencias o conductas particulares de naturaleza instrumental; por ejemplo, si se dice, Juan está hablando, se dice que lo hace como conducta instrumental que tiene una finalidad, como sería entablar una plática con otra persona. En el mismo tenor el autor hace mención de acontecimientos, ocurrencias o conductas particulares cuando alude a reír, tirar besos, abrazar a alguien, comer, caminar, etcétera.
}

siciones que se aplican a conjuntos o colecciones de ocurrencias, sino a ocurrencias o conductas particulares.

Adicionalmente, ¿cuándo es que al afrontamiento, la empatía, la religiosidad y el optimismo se les inscribieron dentro de las categorías de tendencia? Si, como recién se dijo, afrontamiento en algún sentido podría formar parte de las capacidades, en un sentido opuesto ni empatía ni religiosidad pueden concebirse como fenómenos o conceptos psicológicos, y por consiguiente, de ambos no se podría asegurar que son tendencias.

Confiamos que la cita de Ryle permitirá al lector dejar claro lo anterior:

Las tendencias ${ }^{6}$ son diferentes de las capacidades y de las propensiones. "Se comportaría así si..." difiere de "podría"; y "lo hace regularmente... cuando...", difiere de "puede". En pocas palabras, decir "puede" es decir que no existe la certeza de que algo no será el caso, mientras que decir "tiende a" equivale a decir que hay una buena probabilidad de que algo será, o fue, el caso. Así, "tiende a" implica "puede" pero no es implicado por "puede" (Ryle, 1967; p. 117).

Se puede ser empático hoy pero no mañana, porque dependerá del momento y la circunstancia en que entienda y comparta los estados emocionales de otras personas. Una persona no transita por la vida siendo empática de manera consistente, esto es, a lo largo del tiempo y entre situaciones, que son justo las características que distinguen a las palabras o expresiones con que se hace alusión a las tendencias (i.e., personalidad). De nueva cuenta, una confusión lógica trae consigo una confusión conceptual, que trae consigo una confusión metodológica y, finalmente, una confusión interpretativa. De ahí que se pregunte ahora, ¿̇en qué consiste el factor subyacente conocido como resiliencia en este instrumento? Atendiendo a la multiplicidad de factores o dimensiones, algunos de los cuales podrían eventualmente decirse que forman parte de la fenomenología de lo psicológico, pero otros en definitiva no, lo único que queda claro es que la disposición o el factor subyacente llamado resiliencia es todo aquello que uno o más autores quieran decir que es; ni más, ni menos.

Notará el lector que de todos los factores o dimensiones incluidos en la Tabla 3, a una buena parte no se le puede ubicar dentro de una categoría general de análisis de y sobre lo psicológico, ni inclusive dentro de una de lo social; otros, los propiamente psicológicos, incluyen tanto a capacidades, inclinaciones o propensiones como a sentimientos. Por consiguiente, ¿cómo es que un concepto, el de resiliencia, definido por los autores como capacidades adaptativas, puede incluir a factores o dimensiones de tan diversa nomenclatura? En última instancia, se pregunta, ¿cuál es, pues, la utilidad, pertinencia o ventaja de un concepto ajeno a la psicología, que carece de identidad conceptual propia y que arropa en su contenido a cuantos factores o dimensiones incluyan los autores, al margen de si son o no psicológicos?

\footnotetext{
${ }^{6}$ Las palabras en negritas son del autor del presente trabajo.
} 
Permítasenos plantear un reto al lector, que se hará en forma de pregunta, aclarándole por anticipado que lo hacemos en un tono respetuoso y exento de tintes eufemistas: qué pensaría si el autor de este trabajo, dando continuidad a estas prácticas tan sui generis de nuestra psicología contemporánea, asegurase lo siguiente. Habida cuenta que existe una conocida condición de vulnerabilidad biológica llamada "malnutrición", 7 intempestivamente adopta el término, lo redefine y argumenta teóricamente que existe la malnutrición psicológica. En principio, es posible que el lector se diga a sí mismo que el autor está haciendo un uso metafórico del concepto de malnutrición. Acto seguido el autor se propone "medir" a la malnutrición psicológica con base en un instrumento que incluiría los siguientes 12 factores o dimensiones, i.e., competencias intrapersonales "psíquicas", habilidades motoras, afrontamiento ante el estrés psico-bio-sociocultural, sentido del humor, etcétera.

Una vez diseñado el instrumento, que consta de 120 preguntas (10 por factor o dimensión), el autor lo somete a las pruebas estadísticas de rigor para probar sus propiedades psicométricas. En el supuesto de que al final del proceso todos los indicadores de bondad del ajuste fueran los adecuados, ¿podría el lector poner en entredicho o en tela de duda la pertinencia del concepto de malnutrición psicológica y de los factores o dimensiones subyacentes?

Si lo pensó, esbozó al menos una sonrisa y se cuestionó la pertinencia del concepto, del instrumento y de esos resultados, me permito felicitarlo, está en buen camino de iniciar, si no lo ha hecho aún, una lucha ardua, difícil, con enormes contratiempos, pero a la vez necesaria y urgente: en contra de esas prácticas sui generis, muy propias de quienes conciben que la psicología y lo psicológico puede ser cualquier cosa que se tenga a mano, y por qué no, hasta lo que se pueda inventar; lo que significa que hay que luchar en favor de una psicología con identidad propia. Y me permito felicitarlo, asimismo, porque con toda seguridad habrá entendido que el modelamiento con ecuaciones estructurales es una herramienta metodológica de apoyo; no es la quintaesencia ni la varita mágica con la que se resolverán los problemas teóricos y conceptuales de la psicología contemporánea (se recomienda la lectura de Burgos, 2001).

De lo contrario, de manera infortunada, aceptará que existe la malnutrición psicológica, que también existen fenómenos tales como el de la hipertensión psicológica, la inmunología perceptivo-afectivo-conductual y el de ciclo vital psico-endocrinológico-social. Eventualmente adaptará el instrumento, realizará investigación y propondrá programas de intervención para abatir la malnutrición psicológica, eso sí, todo en el nombre de la ¡ciencia! Quizá se interese hasta en formar parte del grupo de psicólogos que elaboren la teoría de la malnutrición psicológica. Sin embargo, es menester aclararle a este segundo lector que el concepto, el instrumen-

\footnotetext{
${ }^{7}$ Que se define como un estado en el que la condición física se degrada, limitando un nivel adecuado de desempeño en las diferentes esferas de la vida.
}

to y sus factores, así como los resultados obtenidos, no fueron sino producto de la imaginación del autor.

\section{A manera de conclusiones}

El análisis conceptual constituye una herramienta metodológica de apoyo en la psicología, aun cuando en general se le suele desestimar por el colectivo de psicólogos. Ello ha traído consigo que con frecuencia se pase por alto la importancia de conocer de dónde provienen los términos (del lenguaje ordinario o si son propiamente técnicos), sino también de ser competentes al momento de plantear su correcta definición y su posterior medición. Sobre esto último, la historia reciente en nuestra disciplina nos ha dado muestras fehacientes de cómo la confusión lógica que subyace a los planteamientos teóricos de muchos autores se traduce en una confusión conceptual, en otra metodológica y, finalmente, en una de interpretación. Ejemplos sobre el respecto abundan en la literatura: inteligencia, personalidad, motivación, autoeficacia y resiliencia; son unos cuantos que han perpetuado estas confusiones consecutivas en las que suelen incurrir los autores (véase Ribes, 1989, 2004, 2010), sin que necesariamente se percaten de sus propias inconsistencias discursivas en torno a la fenomenología de aquello que se presume es lo psicológico. Aquí se ha analizado al último de esos términos, evidenciando que a la par que se trata de un término proveniente del lenguaje ordinario — que por lo demás e injustificadamente se le ha definido como concepto, esto es, como si fuera un término técnico-, es ajeno a la propia psicología.

Cuando Ribes (1990) denunció que la psicología se había convertido en una Torre de Babel, con esto aseguraba que cada psicólogo le confería a los términos un significado distinto, propiciando que no sólo fuera víctima de una confusión conceptual, sino también de una terminológica. Si el de resiliencia, tal y como se ha analizado a lo largo de este trabajo, no es un término que se refiere o aplica a la fenomenología de lo psicológico, no se puede por ningún motivo y bajo ninguna circunstancia legitimar su uso, sin antes entender que un análisis conceptual es requisito sine qua non para eliminar los errores conceptuales y terminológicos que han dominado en la psicología, en particular la del siglo XX. Sin temor a equivocarnos, diríamos que es tiempo ya de cerrar la Caja de Pandora. De otra suerte, como se propuso al final del apartado previo, ¿quién podría cuestionar al autor de este trabajo la existencia, utilidad y pertinencia de conceptos o expresiones como el de malnutrición psicológica?

Permítasenos concluir citando a Ribes (1990) en extenso: Analizar funcionalmente el habla ordinaria no sólo permite derruir viejas mitologías, sino que también procura un primer deslinde de los diversos tipos de eventos y procesos que conforman el comportamiento humano. Este deslinde conceptual se vuelve prioritario por dos razones: primero, porque permite advertir que no existen correspondencias unívocas entre términos y expresiones del lenguaje ordinario y tipos de procesos o 
eventos psicológicos: un mismo término tiene significaciones funcionales diversas $[\ldots]$; segundo, porque a partir de la delimitación funcional del uso de los términos "mentales" en el lenguaje ordinario, es posible construir un lenguaje técnico que

\section{Referencias}

Becoña, E. (2006). Resiliencia: Definición, características y utilidad del concepto. Revista de Psicopatología y Psicología Clínica, 11, 125-146.

Burgos, J. E. (2001). Comentario: Variables latentes, conceptos y definiciones. Acta Comportamentalia, 9, 251-275.

Castillo, R. D. y Haz, A. M. (2003). Adultos resilientes al maltrato físico en la infancia. Terapia Psicológica, 21, 105-119.

Connor, K. M., \& Davidson, J. R. T. (2003). Development of a new resilience scale: The Connor-Davidson Resilience Scale (CD-RISC). Depression and Anxiety, 18, 76-82.

Cornejo, W. (2010). Resiliencia: Una definición. Revista Electrónica Athenea, 2. (Recuperado el 9 de abril de 2013, de htpp://www.academiaperuanadepsicología.org).

Earvolino-Ramirez, M. (2007). Resiliencie: A conceptual analysis. Nursing Forum, 41, 73-82.

Fernández-Lansac, V. y Crespo, M. (2011). Resiliencia, personalidad resistente y crecimiento en cuidadores de personas con demencia en el entorno familiar: Una revisión. Clínica y Salud, 22, 21-40. DOI: $10.593 / \mathrm{c} 12011 \mathrm{v} 22 \mathrm{n} 1 \mathrm{a} 2$

García-Cadena, C. H. (2009). Cómo investigar en psicología. México: Trillas.

Garcia-Dia, M. J., DiNapoli, J. M., Garcia-Ona, L., Jakobowski, R., \& O'Flaherty, D. (2013). Conceptual analysis: Resiliencie. Archives of Psychiatric Nursing, 27, 264-270. DOI: 10.1016/j.apnu.2013.07.003.

Gaxiola, J. C. (2013). Aportaciones conceptuales al estudio de la resiliencia. En J. C. Gaxiola y J. Palomar (Coords.), Estudios de resiliencia en América Latina. Volumen 2 (pp. 1-17). México: Universidad de Sono$\mathrm{ra} /$ Universidad Iberoamericana/Pearson.

Gaxiola, J. C. y Frías, M. (2007). Los factores protectores y la adaptabilidad al abuso infantil: Un estudio con madres mexicanas. Revista Mexicana de Psicología, 24 (Número Especial), 222-223.

Gaxiola, J. C., Frías, M., Hurtado, M. F., Salcido, N. L. y Figueroa, F. (2011). Validación del Inventario de Resiliencia (IRES) en una población del noroeste de México. Enseñanza e Investigación en Psicología, 16, 73-83.

Gaxiola, J. C., González, S., Contreras, Z. y Gaxiola, E. (2012). Predictores del rendimiento académico con disposiciones resilientes y no resilientes. Revista de Psicología, 30, 48-74.

Gaxiola, J. C., Pérez, R. F. y González, S. (2013). Resiliencia, autorregulación, factores protectores y calidad de vida en personas con diabetes mellitus tipo II e hipertensión arterial. En J. C. Gaxiola y J. Palomar (Coords.), Estudios de resiliencia en América Latina. Volumen 2 (pp. 107120). México: Universidad de Sonora/Universidad Iberoamericana/Pearson.

González-Arratia, N. I., Reyes, M., Valdez, J. L. y González, S. (2011). Resiliencia, autoestima y personalidad resistente en niños y adolescentes con antecedentes de maltrato. Revista Mexicana de Investigación en Psicología Socialy de la Salud, 1, 51-62.

González-Arratia, N. I., Valdez, J. L. y González, S. (2011). Investigación en resiliencia: ¿Qué hemos aprendido? En J. Moral de la Rubia, J. L. Valdez y N. I. González-Arratia (Eds.), Psicología y salud (pp. 157-172). Coahuila, México: Consorcio de Universidades Mexicanas.

González-Arratia, N. I., Valdez, J. L., Oudhof, H. y González, S. (2012). Resiliencia y factores protectores en menores infractores y en situación de calle. Psicología y Salud, 22, 49-62.

Ibáñez, C. (1992). Medición de aptitudes intelectuales mediante procedimientos interactivos. Revista Mexicana de Análisis de la Conducta, 18, 103 139.

Noriega, M. G., Angulo, B. y Hernández, R. C. (2011). La resiliencia: Un tema de hoy. Perspectivas Docentes, 45, 52-55. tenga correspondencia unívoca con niveles equivalentes de significación funcional en dicho lenguaje (p. 19).

Palomar, J., Matus, G. L. y Victorio, A (2012). ¿De qué está hecha la resiliencia de pobres extremos del centro de México? Anuario de Psicología Clínica y de la Salud, 8, 59-74.

Quiceno, J. M., Vinaccia, S., Barrera, Y. A., Latorre, R., Molina, D. C. y Zubieta, F. A. (2013). Resiliencia, felicidad, depresión, ideación suicida y afrontamiento del estrés en estudiantes universitarios colombianos. En J. C. Gaxiola y J. Palomar (Coords.), Estudios de resiliencia en América Latina. Volumen 2 (pp. 71-88). México: Universidad de Sonora/Universidad Iberoamericana/Pearson.

Real Academia Española (2011). Avances de la 23a edición del Diccionario de la Lengua Española. Madrid: Autor.

Restrepo, C. (2009). Anteproyecto de investigación: evaluación de la calidad de vida, la resiliencia y la depresión en adolescentes escolarizados de la ciudad de Medellín. Manuscrito no publicado. Departamento de Psicología, Universidad de San Buenaventura, Medellín, Colombia.

Restrepo, C., Vinaccia, S. y Quiceno, J. M. (2011). Resiliencia y depresión: Un estudio exploratorio desde la calidad de vida en la adolescencia. Suma Psicológica, 18, 41-48.

Ribes, E. (1989). La inteligencia como comportamiento: Un análisis conceptual. Revista Mexicana de Análisis de la Conducta, 15, 51-67.

Ribes, E. (1990). Psicología general. México: Trillas.

Ribes, E. (2004). Behavior is abstraction not ostension: Conceptual and historical remarks on the nature of psychology. Behavior and Philosophy, 32, $55-68$.

Ribes, E. (2006). Competencias conductuales: Su pertinencia en la formación y práctica profesional del psicólogo. Revista Mexicana de Psicología, 23, 19 26.

Ribes, E. (2010). Lenguaje ordinario y lenguaje técnico: Un proyecto de currículo universitario para la psicología. Revista Mexicana de Psicología, 27, 55-64.

Ribes, E. (2011). El concepto de competencia: Su pertinencia en el desarrollo psicológico y la educación. Bordón, 63, 33-45.

Roca, J. (2001). Sobre el concepto de "proceso" cognitivo. Acta Comportamentalia, 9, 21-30.

Rodríguez, M., Pereyra, M. G., Gil, E., Jofré, M., de Bortoli, M. y Labiano, L. M. (2009). Propiedades psicométricas de la escala de resiliencia versión argentina. Evaluar, 9, 72-82.

Ruiz, G. T. y López, A. E. (2012). Resiliencia y dolor crónico. Estudios de Psi cologia, 5, 1-11.

Ryle, G. (1967). El concepto de lo mental. Buenos Aires: Paidós.

Saavedra, E. y Villalta, M. (2008). Medición de las características resilientes, un estudio comparativo en personas entre 15 y 65 años. Liberabit, 14, 31-40.

Serrano-Parra, M. D., Garrido-Abejar, M., Notario-Pacheco, B., BartoloméGutiérrez, R., Solera-Martínez, M. y Martínez-Vizcaino, V. (2012). Validez de la escala de resiliencia de Connor-Davidson (CD-RISC) en una población de mayores entre 60 y 75 años. International Journal of Psychological Research, 5, 49-57.

Vinaccia, S., Quiceno, J. M. y Moreno-San Pedro, E. (2007). Resiliencia en adolescentes. Revista Colombiana de Psicologia, 16, 139-146.

Wagnild, G. M., \& Young, H. M. (1993). Development and psychometric evaluation of the resilience scale. Journal of Nursing Measurement, 1, 165178.

Windle, G. (2011). What is resilience? A review and concept analysis. Reviews in Clinical Gerontology, 21, 152-169. DOI: 10.1017/S0959259810000420.

(Artículo recibido: 11-11-2013; revisado: 25-06-2014; aceptado: 21-07-2014 